\title{
Illumination-induced changes of the Fermi surface topology in three-dimensional superlattices
}

\author{
N. A. Goncharuk, L. Smrčka, P. Svoboda, P. Vašek, and J. Kučera \\ Institute of Physics, Academy of Science of the Czech Republic, v.v.i., \\ Cukrovarnická 10, 162 53 Praha 6, Czech Republic \\ Yu. Krupko \\ Grenoble High Magnetic Field Laboratory CNRS, \\ 25 Avenue des Martyrs B.P.166, 38042 Grenoble cedex 9, France \\ W. Wegscheider \\ University of Regensburg, Universitästrasse 31, D-93040 Regensburg, Germany
}

(Dated: November 1, 2018)

\begin{abstract}
The magnetoresistance of the MBE-grown $\mathrm{GaAs} / \mathrm{Al}_{0.3} \mathrm{Ga}_{0.7} \mathrm{As}$ superlattice with Si-doped barriers has been measured in tilted magnetic fields in the as-grown state, and after brief illumination by a red-light diode at low temperature, $T \approx 0.3 \mathrm{~K}$. A remarkable illumination-induced modification of magnetoresistance curves has been observed, which indicates a significant change of the superlattice Fermi surface topology. Analysis of magnetoresistance data in terms of the tight-binding model reveals that not only electron concentration and mobility have been increased by illumination, but also the coupling among 2D electron layers in neighboring quantum wells has been reduced.
\end{abstract}

PACS numbers: 73.43.Qt; 73.43.Jn; 02.60.Ed; 03.65.Sq

\section{INTRODUCTION}

Semiconductor superlattices (SLs), first proposed by Esaki and Tsu in $1970^{\underline{1}}$, are layered structures composed of regularly spaced quantum wells (QWs) separated by barriers. A review of early research of SLs was given, e.g., by $\mathrm{Maan}^{2}$ and $\mathrm{Helm} \cdot \underline{\underline{3}}$

When doped by donors, the barriers provide electrons for two-dimensional (2D) electron sheets inside the QWs. As discussed, e.g., in Refs, $\frac{4.5}{\underline{5}}$ the resulting electronic structure of the SL depends on the strength of the periodic potential as well as on the level of the doping, and can range from a quasi-three-dimensional (3D) system to a nearly $2 \mathrm{D}$ system of independent layers of $2 \mathrm{D}$ electron gas in each QW.

For strong potential, usually provided by thick and high barriers, the tunnel-coupling of electron layers, associated with overlap of wave functions from neighboring wells, is negligible and SL minibands reduces to almost discrete energy levels. Moreover, in the narrow wells, the minibands are well separated and usually only one miniband is occupied. Then the electron system can be considered as 2D, and the Fermi surface (FS) is cylindrical in shape.

With decreasing the barrier thickness tunneling of electrons becomes increasingly important, manifesting itself in the broadening of minibands. The FS becomes the corrugated cylinder if the Fermi energy $E_{F}$ is above the miniband top, or closed semielliptical Fermi ovals (each fully contained within the first Brillouin zone) for the Fermi energy below the miniband top. The latter electronic structure corresponds to the anisotropic 3D electron gas. The development of the FS topology with increasing tunnel-coupling between QWs is schematically shown in Fig. 1. We call the change of the FS from an open corrugated cylinder towards closed ovals the $2 \mathrm{D} \rightarrow 3 \mathrm{D}$ transition.

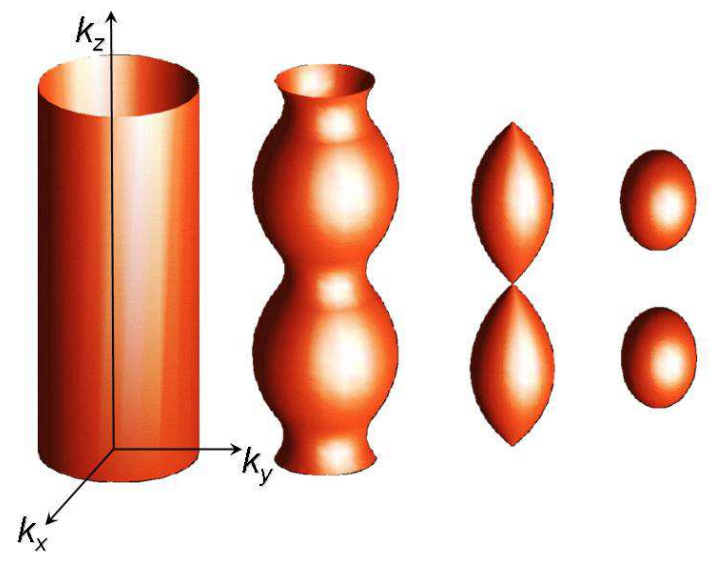

FIG. 1: (Color online) The scheme of a variety of SL Fermi surfaces. From the left to the right: a cylinder is related to $E_{F}$ lying well above the narrow SL miniband top (strictly $2 \mathrm{D}$ behavior); a corrugated cylinder corresponds to $E_{F}$ lying above the SL miniband top (2D behavior); touching FSs arise when $E_{F}$ lies at the top of the SL miniband and define the point of the $2 \mathrm{D} \rightarrow 3 \mathrm{D}$ transition; separated ovals occur when $E_{F}$ lies below the SL miniband top (strictly 3D behavior).

The SL Fermi surface topology can be determined experimentally by magnetotransport measurements in magnetic fields $\vec{B}$ tilted with respect to the $\mathrm{QW}$ planes. ${ }^{4.5 .6}$ The quasiclassical theory predicts that an electron is driven by the Lorentz force around orbits, defined in $k$-space by intersections of the FS and planes 
perpendicular to the direction of the applied magnetic field. In the case of closed orbits, the electron motion is quantized into Landau levels, that manifest themselves through Shubnikov-de Haas ( $\mathrm{SdH}$ ) oscillations observable in the longitudinal magnetoresistance.

The Onsager-Lifshitz relation ${ }^{7}$ states that $\mathrm{SdH}$ oscillations are periodic in $1 / B$ with the period determined by the extremal cross-sections of the FS. $\stackrel{8}{=}$ By tilting the sample, a number of cross-sections can be examined and the FS reconstructed. This is true only for closed FSs. For corrugated cylinders, open orbits appear for field orientations close to the QW planes, and the $\mathrm{SdH}$ oscillations are suppressed.

An interesting case are SLs with the Fermi energy just below the top of the miniband. The Fermi ovals nearly touch the Brillouin zone boundaries and, therefore, the extremal closed orbits corresponding to the in-plane magnetic fields also nearly touch each other. If the magnetic field is strong enough, the Onsager-Lifshitz quantization scheme can be violated by the magnetic breakdown, and the closed orbits effectively interconnected to the open ones. In that case we can speak about magnetic-fieldinduced $3 \mathrm{D} \rightarrow 2 \mathrm{D}$ transition. At this point the quasiclassical description fails and the quantum-mechanical treatment becomes necessary.

The deviations from the standard $1 / B$ periodicity due to the magnetic breakdown have been already reported in Ref. 4, for SdH oscillations corresponding to the Landau levels with low indices, measured in strong tilted magnetic fields. The magnetic-field-induced $3 \mathrm{D} \rightarrow 2 \mathrm{D}$ transition was intentionally studied by Jaschinski et al $\underline{\underline{\underline{9}}}$ Our experimental investigation of this effect is described in Ref. 10 and interpreted by the full quantum-mechanical theory developed in Ref. 11 .

In the present publication we concentrate on another possibility: the illumination-induced changes of the superlattice FS topology. The illumination of doped semiconductor structures at low temperatures leads to the persistent photoconductivity effect (PPC) and increases the concentration of free carriers. As the increase of the concentration means the increase of the Fermi energy, one expects that it might be possible to change the FS topology from closed ovals to a corrugated cylinder. This paper reports on the experimental study demonstrating that the illumination of the SL sample can indeed change the electronic structure of the SL and induce a transition from a $3 \mathrm{D}$ to $2 \mathrm{D}$ state. To exclude the magnetic breakdown, we limit our experiments to lower magnetic fields, for which the quasiclassical interpretation of magnetotransport measurements is valid.

\section{EXPERIMENT}

The sample employed in our experiments is the MBEgrown SL (30 periods), which consists of $29 \mathrm{GaAs}$ QWs of width $d_{w}=4.5 \mathrm{~nm}$, separated by $4 \mathrm{~nm}$ wide $\mathrm{Al}_{0.3} \mathrm{Ga}_{0.7} \mathrm{As}$ barriers. This gives the period $d_{z}$ of the SL equal to
$8.5 \mathrm{~nm}$. Each barrier is composed of the central Si-doped part $2.7 \mathrm{~nm}$ thick, which is flanked by $0.65 \mathrm{~nm}$ thick undoped spacer layers on both sides. The samples from the same wafer were used in our previous study of the magnetic-field-induced $3 \mathrm{D} \rightarrow 2 \mathrm{D}$ transition ${ }^{10}$ in magnetic fields up to $26 \mathrm{~T}$.

The Hall bar samples have been patterned by means of the optical lithography, equipped with evaporated $\mathrm{Au}$ GeNi contact pads and adjusted to ceramic chip carriers. The width of their conducting channel was $w=400 \mu \mathrm{m}$, the length of the sample was $L=1000 \mu \mathrm{m}$. The chip carrier with the sample has been attached to a rotation plate in a ${ }^{3} \mathrm{He}$ cryostat, that fitted the bore of a superconducting magnet providing magnetic fields from 0 to $13 \mathrm{~T}$, the range of fields for which the quasiclassical interpretation of $\mathrm{SdH}$ oscillation is reasonable $\underline{\underline{10}}$

The plate made it possible to rotate the sample to any angle between the perpendicular $\left(\varphi=0^{\circ}\right)$ and in-plane $\left(\varphi=90^{\circ}\right)$ field orientations. Standard lock-in technique at $f=13 \mathrm{~Hz}$ has been employed to measure simultaneously both the longitudinal $\rho_{x x}(B)$ and Hall $\rho_{x y}(B)$ resistances during sweeping the magnetic field up or down. All the data presented below were taken at the temperature of about $0.3 \mathrm{~K}$.

A red-light LED was installed next to the sample holder to facilitate an "in situ" illumination of the cooleddown sample in order to enhance the electron concentration and induce the PPC. Short current pulses $(\approx$ $100 \mathrm{msec}$ ) were subsequently applied to the LED and the decrease of the zero-field resistance of the sample due to the illumination was monitored. A few such pulses were sufficient to get the resistance saturation. All the curves reported below for the "illuminated" sample have been measured in this saturated state.

\section{THEORY: MODEL CALCULATIONS}

\section{A. Tight-binding superlattice miniband}

It follows from the general consideration presented in the introduction that the energy spectrum of the SL can be written as

$$
E(\vec{k})=\frac{\hbar^{2} k_{x}^{2}}{2 m^{*}}+\frac{\hbar^{2} k_{y}^{2}}{2 m^{*}}+E\left(k_{z}\right),
$$

where $m^{*}=0.067 m$ denotes the effective mass of electrons moving freely in the $(x, y)$ plane of the QWs, and $E\left(k_{z}\right)$ is the dispersion relation of the miniband which describes tunneling of electrons through the barriers. $E\left(k_{z}\right)$ is a periodic function, $E\left(k_{z}\right)=E\left(k_{z}+2 \pi / d_{z}\right)$. Together with the knowledge of $E_{F}$, Eq. (1D) determines the shape of the SL Fermi surface.

The aim of this publication is to determine the FS of the SL from measured periods of SdH oscillations in tilted magnetic fields. Therefore we employ the simplest possible model of $E\left(k_{z}\right)$ to minimize the number of fitting parameters describing the miniband structure. 
The natural choice is the tight-binding model in which

$$
E\left(k_{z}\right)=-2 t \cos \left(k_{z} d_{z}\right)
$$

where $t$ is the coupling constant, associated with the matrix element of the SL potential between the overlapping wave functions from neighboring wells, which determines the miniband width. The SL growth parameters such as the effective barrier thickness, the barrier height and the QW width are thus represented by this single constant ${ }^{12}$, and the shape of the FS is described by three parameters $t, d_{z}$ and $E_{F}$. It will be shown in subsequent sections that this approximation of $E\left(k_{z}\right)$ yields a reasonable agreement with experimental findings.

The cosine approximation for a single SL miniband gives the following expressions for the electron concentration per layer:

$$
N=\frac{m^{*}}{\pi^{2} \hbar^{2}}\left[E_{F} \arccos \left(-\frac{E_{F}}{2 t}\right)+\sqrt{4 t^{2}-E_{F}^{2}}\right],
$$

for a closed FS and

$$
N=\frac{m^{*}}{\pi \hbar^{2}} E_{F}
$$

for an open FS. These expressions include the spin degeneracy. According to Eqs. (3)-(4), the knowledge of the electron concentration, which can be determined from the Hall magnetoresistance data, allows the prediction of the Fermi energy value $E_{F}$.

\section{B. Quasiclassical approach, perpendicular and tilted magnetic fields}

After application of an external magnetic field $\vec{B}$, the energy spectrum is converted to a set of Landau levels. The orbits corresponding to extremal cross-section areas, $A_{k}$, of the FS are most important. According to the Onsager-Lifshitz quantization rule ${ }^{7}$, the expression

$$
A_{k}=\frac{2 \pi|e| B}{\hbar}\left(n+\frac{1}{2}\right), \quad n=0,1,2, \cdots,
$$

determines a set of magnetic fields at which Landau levels cross the Fermi energy.

We have chosen the geometry where a uniform magnetic field applied in the $(y, z)$ plane $\vec{B}=$ $(0, B \sin \varphi, B \cos \varphi)$ is tilted by an angle $\varphi$ with respect to the growth axis $z$, and its orientation varies between perpendicular $\left(B=B_{z}, \varphi=0^{\circ}\right)$ and in-plane $\left(B=B_{y}, \varphi=90^{\circ}\right)$ configurations.

The relation between the period of $\mathrm{SdH}$ oscillations in reciprocal magnetic fields, $\Delta(1 / B)$, and $A_{k}$ can be written as

$$
A_{k}=\frac{2 \pi|e|}{\hbar \Delta(1 / B)}
$$

With the energy spectrum given by Eqs. (1) and (2), the extremal cross-section area in tilted magnetic fields takes the form

$$
\begin{aligned}
& A_{k}^{\text {tilted }}(\varphi)=\frac{\sqrt{2 m^{*}}}{\hbar} \\
& \quad \times 2 \int \sqrt{E_{F}-\frac{\hbar^{2} k^{\prime 2} \cos ^{2} \varphi}{2 m^{*}}+2 t \cos \left(k^{\prime} \sin \varphi d_{z}\right)} d k^{\prime}
\end{aligned}
$$

where

$$
k^{\prime}=\frac{k_{y}}{\cos \varphi}-k_{z 0} \tan \varphi=k_{z 0} \cot \varphi-\frac{k_{z}}{\sin \varphi} .
$$

In Eq. (8) $k_{z 0}$ is the point at which the plane perpendicular to the direction of the magnetic field cuts the axis $k_{z}$. Two types of extremal cross-sections exist: $A_{k}^{B}$ for $k_{z 0}=0$ corresponds to a "belly" orbit and $A_{k}^{N}$ for $k_{z 0}= \pm \pi / d_{z}$ to a "neck" orbit. A wide range of tilt angles $\varphi$ makes it possible to determine the FS of the sample.

In strictly perpendicular magnetic fields, the "belly" and "neck" extremal orbits become circular, with crosssection areas given by simple expressions

$$
\begin{aligned}
& A_{k}^{B}=\frac{2 m^{*} \pi}{\hbar^{2}}\left(E_{F}+2 t\right), \\
& A_{k}^{N}=\frac{2 m^{*} \pi}{\hbar^{2}}\left(E_{F}-2 t\right) .
\end{aligned}
$$

For the closed FSs, $\left|k_{z}\right|$ is always less than $\pi / d_{z}$, and only "belly" orbits with areas $A_{k}^{B}$ occur.

It follows from Eq. (5) that in the perpendicular magnetic field the quantized energies corresponding to either the "belly" or "neck" orbit cut the Fermi energy at magnetic fields given by equations

$$
\begin{aligned}
& \frac{\hbar|e| B^{B}}{m^{*}}\left(n+\frac{1}{2}\right)=E_{F}+2 t, \\
& \frac{\hbar|e| B^{N}}{m^{*}}\left(n+\frac{1}{2}\right)=E_{F}-2 t .
\end{aligned}
$$

Note that we use the energy scale with the origin in the middle of the SL miniband, i.e., the miniband bottom lies at $E=-2 t$ and its top at $E=2 t$.

\section{EXPERIMENTAL RESULTS}

Experimental data collected for the SL before illumination are displayed in Fig. 2, Due to quasi-3D nature of its electronic structure, quantum Hall plateaus are not distinguished on Hall magnetoresistance curves $\rho_{x y}$. The spin unresolved $\mathrm{SdH}$ oscillations can be noticed on all $\rho_{x x}$ curves, including those obtained in strictly in-plane magnetic fields. This confirms beyond question that the FS is a closed semielliptic oval. The oscillations have been found to be periodic in $1 / B$ and a single period is well detectable for all angles. 


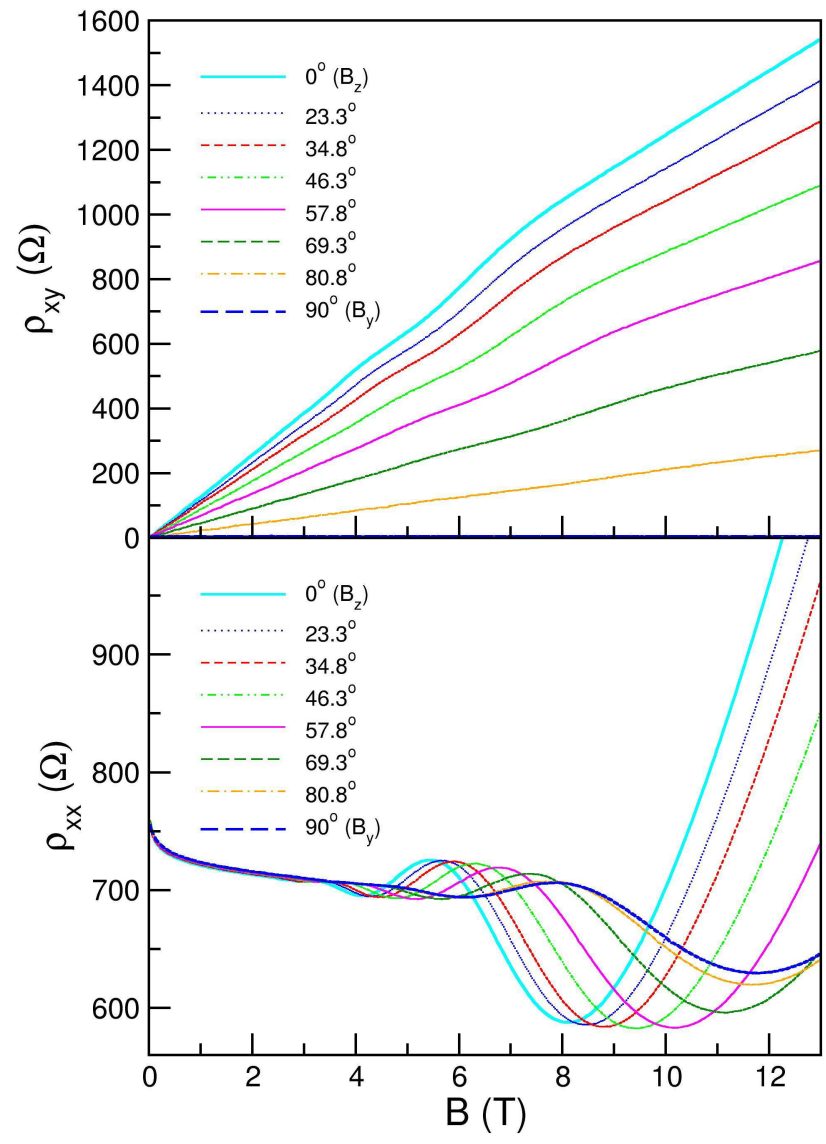

FIG. 2: (Color online) The experimental curves of the transversal $\rho_{x y}$ and longitudinal $\rho_{x x}$ magnetoresistance measured at $T=0.3 \mathrm{~K}$ for the set of tilt angles $\varphi$ of the applied magnetic field on the SL sample "in dark".

Knowing the number of QWs in the SL, we can determine the electron concentration per 2D layer. It follows from the Hall magnetoresistance measured in low perpendicular magnetic fields (under $3 \mathrm{~T}$ ), that $N_{d, H} \simeq$ $1.656 \times 10^{11} \mathrm{~cm}^{-2}$.

The experimental periods deduced from the SdH oscillations were put into Eq. (6), to calculate the angular dependence of the extremal cross-section area $A_{k}^{B}(\varphi)$. The nonlinear fitting of Eq. (7) to the experimentally determined curve gives the parameters of the tight-binding model. Excellent fit is possible with values $t=4.6 \mathrm{meV}$, $E_{F}=5.3 \mathrm{meV}$. This implies that the SL miniband width is $4 t=18.4 \mathrm{meV}$ and that the $E_{F}$ lies $3.9 \mathrm{meV}$ bellow the top of the miniband.

The fitted values can be used in Eq. (3) to calculate the electron concentration from the period of the $\mathrm{SdH}$ oscillations. We get $N_{d, S d H} \simeq 1.7 \times 10^{11} \mathrm{~cm}^{-2}$ in very good agreement with $N_{d, H}$. The mobility calculated from the zero-field resistance and the electron concentration reads $\mu_{d}=1.7 \times 10^{3} \mathrm{~cm}^{2} / \mathrm{Vs}$.

Illumination changes dramatically both $\rho_{x x}$ and $\rho_{x y}$ magnetoresistance curves, as compared to those measured "in dark" (see Fig. [3). Both curves became strongly

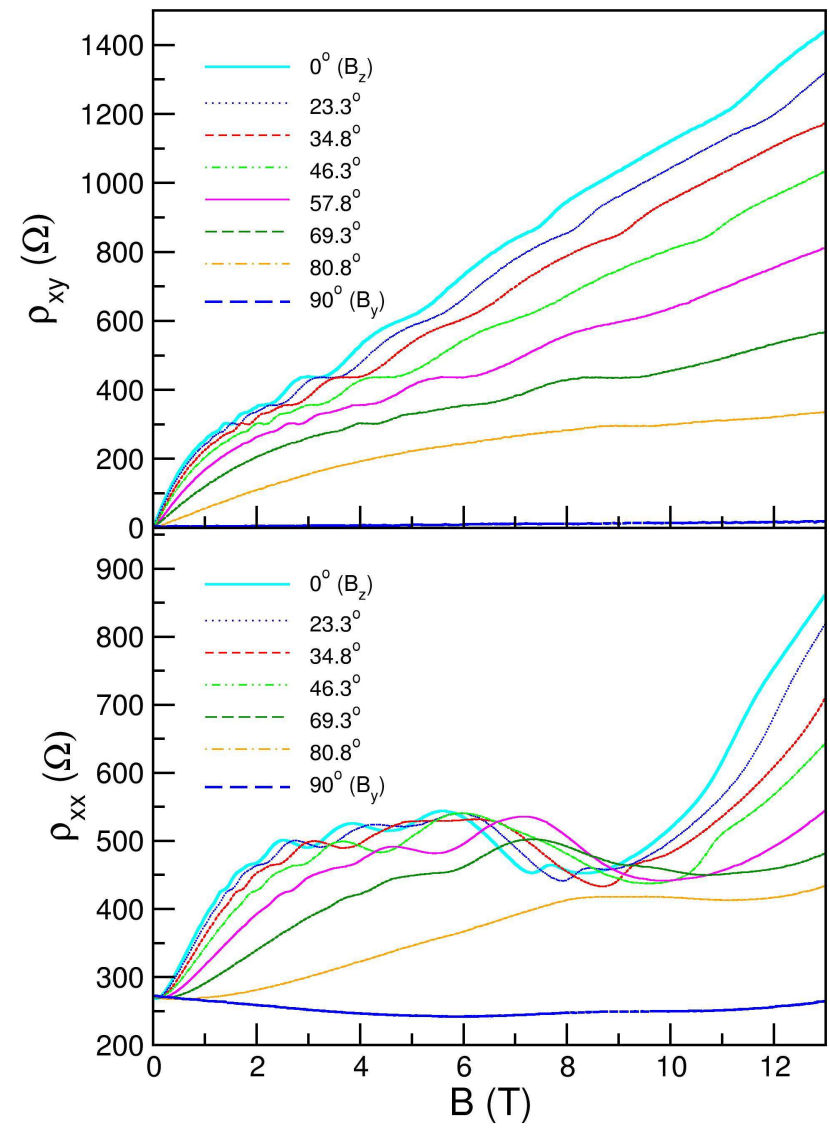

FIG. 3: (Color online) The experimental curves of the transversal $\rho_{x y}$ and longitudinal $\rho_{x x}$ magnetoresistance measured at $T=0.3 \mathrm{~K}$ for the set of tilt angles $\varphi$ of the applied magnetic field on the illuminated SL sample.

nonlinear in the low-field region, indicating that except of the electrons corresponding to the "belly" orbits, new group of electrons close to the "neck" orbits appeared. The zero-field resistance $\rho_{x x}$ drops to less than one third of the original value. The SdH oscillations acquire more complicated form, exhibiting two distinct periods evidently originated from the "belly" and "neck" orbits. The oscillations become weaker with increasing the tilt angle $\varphi$ and disappear completely in strictly in-plane magnetic fields, as it should be for the corrugated cylinder. Hall plateaux appear in $\rho_{x y}$ curves corresponding to minima of the $\mathrm{SdH}$ oscillations in $\rho_{x x}$.

Due to the nonlinear shape of $\rho_{x y}$ in low magnetic fields, the straightforward determination of the electron concentration $N_{i l, H}$ becomes unreliable. From the smooth part of $\rho_{x y}$ in higher perpendicular magnetic fields (above $3 \mathrm{~T}$ ) we got $N_{i l, H} \simeq 2.2 \times 10^{11} \mathrm{~cm}^{-2}$.

There are two angular dependences of extremal crosssections, corresponding to "belly" and "neck" orbits, determined from two experimental periods of SdH oscillations. We were able to detect oscillation periods only up to $\varphi \approx 35^{\circ}$. Cross-section areas of the "belly" and "neck" orbits grow slowly for $\varphi<\varphi_{c}$, where $\varphi_{c} \approx 60^{\circ}$ 



FIG. 4: (Color online) The experimental and theoretical extremal cross-sections of the SL Fermi surface before (a) and after (b) illumination against the tilt angle $\varphi$ between the sample and the applied magnetic field. The green point on the lower figure emphasizes the position of the critical angle $\varphi_{c}$ at which the "belly" and "neck" extremal cross-section areas become equal. Each inset shows two limiting cross-sections of the FS corresponding to the perpendicular $\left(\varphi=0^{\circ}\right)$ and parallel $\left(\varphi=90^{\circ}\right)$ orientations of the magnetic field with respect to the sample plane. The $k_{\alpha}$ on the horizontal axis denotes either $k_{y}\left(\right.$ for $\left.B=B_{z}, \varphi=0^{\circ}\right)$ or $k_{z}\left(\right.$ for $\left.B=B_{y}, \varphi=90^{\circ}\right)$.

is the critical angle at which both cross-sections become equal $\left(A_{k}^{B}=A_{k}^{N}\right)$. From the experimental angular dependences the parameters of the FS in Eq. (7) can be determined by nonlinear curve fitting. We have found $t=2 \mathrm{meV}, E_{F}=10.5 \mathrm{meV}$, which means that the SL miniband width is $8 \mathrm{meV}$ and that the Fermi energy is $6.5 \mathrm{meV}$ above the top of the miniband.

It follows from this value of $E_{F}$ and Eq. (4) that $N_{i l, S d H} \simeq 2.94 \times 10^{11} \mathrm{~cm}^{-2}$. We consider this number more reliable than $N_{i l, H} \simeq 2.2 \times 10^{11} \mathrm{~cm}^{-2}$, determined from the high-field $\rho_{x y}$ data, which obviously underestimates the true value.

The zero-field resistance and $N_{i l, S d H}$ give the mobility $\mu_{i l}=3.6 \times 10^{3} \mathrm{~cm}^{2} / \mathrm{Vs}$, higher than the mobility $\mu_{d}=$ $1.7 \times 10^{3} \mathrm{~cm}^{2} / \mathrm{V} \mathrm{s}$ of the sample before illumination.

Good agreement between the experimental data, which


FIG. 5: (Color online) (a) Electron energy structure of the SL "in dark" (left figure) and illuminated one (right figure). The Landau levels are plotted against the magnetic field applied perpendicular to the sample. The blue fans are associated with "neck" orbits (and the top of the SL miniband), red ones with "belly" orbits (and the bottom of the SL miniband). The tinged cyan and yellow regions show the SL miniband ranges before and after illumination, respectively. The dashed lines denote the positions of the Fermi energy. (b) The longitudinal magnetoresistance curve $\rho_{x x}$ measured on the SL before (left figure) and after illumination (right figure) in the perpendicular magnetic field. Letters $B$ and $N$ are inscribed above the magnetoresistance oscillation maxima corresponding either to a "belly" or "neck" orbit. (c) The cross-section area of the SL Fermi surface as a function of the perpendicular magnetic field, calculated on the basis of the Onsager-Lifshitz quantization rule given by Eq. (5).

stems from the periods of SdH oscillations, and the theoretical curves based on the cosine approximation of the SL miniband is illustrated in Fig. 4. It confirms that this approach is relevant for description of the electronic structure of the studied sample.

Good consistency of our simple theory with experiments is further confirmed by data obtained in strictly perpendicular magnetic fields, which are presented in Fig. 5. Besides the longitudinal magnetoresistances $\rho_{x x}$, the fans of Landau levels corresponding to the "belly" and "neck" orbits are shown, together with the calculated minibands and Fermi energies of both as-grown and illuminated samples. Intersects of Landau levels with calculated $E_{F}$ coincide with positions of oscillation maxima and thus give the values of cross-section areas which are in excellent agreement with experimental values. The graphical method of comparison of the theory and experiment is very useful as it allows to distinguish whether a peak of the longitudinal magnetoresistance belongs to the "belly" or the "neck" extremal orbit.

Experimentally determined parameters, $t$ and $E_{F}$, have also been used to calculate the density of states (DOS) for several lowest Landau subbands of the SL 



FIG. 6: (Color online) The longitudinal magnetoresistance $\rho_{x x}$ measured on the SL "in dark" (a) and illuminated one (b) with their second derivatives compared to DOSs calculated as functions of the magnetic field applied perpendicular to the sample. The DOSs calculated only for the lowest four eigenenergies are shown.

before and after illumination. The resulting DOSs are shown in Fig. [6 together with corresponding longitudinal magnetoresistance curves and their second derivatives as functions of the perpendicular magnetic field. The reasonable agreement of the oscillation peak positions with the DOS extrema supports our interpretation.

\section{DISCUSSION}

We have measured low-temperature longitudinal and transversal magnetoresistance of the short-period SL with Si-doped barriers in the as-grown state and after illumination.

In the as-grown state, the SL behaves as a 3D system, exhibiting a single period $\mathrm{SdH}$ oscillations for all orientations of the external magnetic field. This implies that the neighboring QWs are strongly coupled and electrons forming an anisotropic 3D gas are present with nonzero probability not only in the QWs but also inside the barriers. It is possible only if all shallow Si donors are ionized and empty shallow levels lie in the thermodynamic equilibrium well above the Fermi energy. Thus the depleted layer is extended through the whole $\mathrm{Al}_{0.3} \mathrm{Ga}_{0.7} \mathrm{As}$ barrier and the Fermi energy is not fixed in $\mathrm{Al}_{0.3} \mathrm{Ga}_{0.7} \mathrm{As}$ by the donor level as in the standard modulation-doped structures.

The situation becomes more complicated after the sample illumination, which leads to the persistent photoconductivity and increases persistently the density of carriers. The origin of the PPC is attributed to ionization of deep $\mathrm{Si}$ donor states in the $\mathrm{Al}_{0.3} \mathrm{Ga}_{0.7} \mathrm{As}$ barrier. As the original "dark" value of the concentration and the magnetoresistance can be recovered by heating the sample to temperatures above $100 \mathrm{~K}$, the illuminated sample is in a reversible metastable state, separated from the thermodynamic equilibrium by the capture barrier which prevents the return of electrons back to ionized Si donor states at low temperatures.

In spite of year-long extensive studies the nature of deep levels and the capture barrier is still a subject of discussions. The detailed discussion of the properties of deep donor levels is beyond the scope of this publication, the main alternative explanations of the PPC and the photoionization process can be found, e.g., in Refs. 13, 14, 15, 16.

Here we consider as important that the capture barrier is formed around the ionized donors inside the doped $\mathrm{Al}_{0.3} \mathrm{Ga}_{0.7} \mathrm{As}$ layer. It can be a combined effect of both the band-offset between $\mathrm{Al}_{0.3} \mathrm{Ga}_{0.7} \mathrm{As}$ and $\mathrm{GaAs}$ and this capture barrier which leads to suppression of the interwell coupling and consequently to the reduction of the miniband width.

\section{CONCLUSIONS}

In conclusion, longitudinal and transversal magnetoresistances have been measured in tilted magnetic fields on the GaAs/AlGaAs SL sample. Illumination of the sample enhances the electron concentration in the QWs and makes the SdH oscillations more complicated. The results have been analyzed in terms of a simple tightbinding model. The effect of the illumination can be described as a $3 \mathrm{D} \rightarrow 2 \mathrm{D}$ transition, where the FS of the system changes from closed ovals contained within the first Brillouin zone to an open corrugated cylinder, characteristic for a system composed of weakly coupled $2 \mathrm{D}$ electron layers. The illumination thus not only enhances the electron concentration in the layers, but simultaneously suppresses the strength of the inter-layer coupling as indicated by the decrease of the SL miniband width. This rather surprising result can be qualitatively explained in terms of increasing the effective barrier height due to a photon-induced ionization of deep donor impurity levels within $\mathrm{Al}_{0.3} \mathrm{Ga}_{0.7}$ As barriers. 


\section{ACKNOWLEDGEMENTS}

This work has been supported by the European Commission contract No. RITA-CT-2003-505474, Ministry of
Education of the Czech Republic Center for Fundamental Research LC510 and Academy of Sciences of the Czech Republic project KAN400100652.
${ }^{1}$ L. Esaki and R. Tsu, IBM Res. Dev. 14, 61 (1970).

2 J. C. Maan, Superlattices and Microstructures 2, No. 6, 557 (1986).

3 M. Helm, Semicond. Sci. Technol. 10, 557 (1995).

${ }^{4}$ L. L. Chang, E. E. Mendez, N. J. Kawai, and L. Esaki, Surface Sci. 113, 306 (1982).

${ }^{5}$ G. Nachtwei, A. Weber, H. Künzel, j. Böttcher, and O. Jaschinski, J. Appl. Phys. 84, 323 (1998).

${ }^{6}$ H. L. Störmer, J. P. Eisenstein, A. C. Gossard, W. Wiegmann, and K. Baldwin, Phys. Rev. Lett. 56, 85 (1986).

7 L. Onsager, Philos. Mag. 43, 1006 (1952).

8 N. W. Ashcroft, N. D. Mermin, Solid State Physics (Saunders, Philadelphia, 1975), p. 368.

9 O. Jaschinski, G. Nachtwei, J. Schoenes, P. Bönsch, and A. Schlachetzki, Physica B, 249-251, 873 (1998).
10 L. Smrčka, P. Vašek, P. Svoboda, N. A. Goncharuk, O. Pacherová, Yu. Krupko, Y. Sheikin, and W. Wegscheider, Physica E 34, 632 (2006).

11 N. A. Goncharuk, L. Smrčka, J. Kučera, and K. Výborný, Phys. Rev. B 71, 195318 (2005).

12 J. Hu and A. H. MacDonald, Phys. Rev. B 46, 12554 (1992).

13 S. B. Zhang and D. J. Chadi, Phys. Rev. B 42, 7174 (1990).

14 J. C. Bourgoin, S. L. Feng, and H. J. von Bardeleben, Phys. Rev. B 40, 7663 (1989).

15 W. Timelthaler, W. Jantsch, and G. Weimann, Semicond. Sci. Technol. 5, 686 (1990).

16 E. P. De Poortere, Y. P. Shkolnikov, and M. Shayegan Phys. Rev. B 67, 153303 (2003). 